\title{
An Iterative Learning Algorithm for Control of an Accelerator Based Free Electron Laser
}

\author{
S. Kichhoff, C. Schmidt, G. Lichtenberg, H. Werner
}

\begin{abstract}
This paper shows the successful application of an iterative learning controller (ILC) to the Free Electron Laser FLASH at DESY, Hamburg, a plant of large international interest for research in physics, chemistry, biology, and engineering. First experimental results demonstrate the applicability of the ILC approach to the low level radio frequency system which controls the electron acceleration.
\end{abstract}

\section{INTRODUCTION}

Free Electron Lasers use linear particle accelerators, which increase the energy of the electrons by interaction with electromagnetic radio frequency (RF) fields [1]. They are operated in pulsed mode, e.g. every second there is a pulse for approximately one millisecond. This pulsed system has the following properties:

- the characteristic disturbances and uncertainties only show small changes from pulse to pulse,

- between pulses, several hundred milliseconds could be used for computing optimal parameters and input signals for the next pulse,

- the FPGA structure of the digital intra pulse controller allows arbitrary input signals at a frequency of $1 \mathrm{MHz}$,

- appropriate models could be identified by standard methods from measurement data.

Thus, an Iterative Learning Control (ILC) strategy appears to be well suited to control this plant [2]. For the application of ILC, the following questions need to be answered [3]:

- which algorithm is appropriate for implementation?

- which type of model is needed by the chosen algorithm?

- how can such models be obtained?

These questions will be addressed in this paper as follows: Section II gives a description of the FEL along with details of the RF system for the linear accelerator with its digital control system, followed by Section III, where a black box model of this RF system is derived. After introducing the Iterative learning control algorithm in Section IV, its implementation on the real time system is described. Finally, experimental results of the first tests are shown in Section V, followed by the conclusions.

\section{The Free Electron LASER FACILITy FLASH}

\section{A. Basic System Structure}

For many research activities a light source that is able to resolve objects on an atomic level would be favorable,

S. Kirchhoff, G. Lichtenberg and H. Werner are with the Institute of Control Systems, Hamburg University of Technology, 21071 Hamburg, Germany, svenspostaweb.de, Lichtenberg@tuhh.de, h. werner@tuhh. de

C. Schmidt is with DESY Hamburg, Notkestrasse 85, 22607 Hamburg, Germany christian.schmidtedesy.de e.g. in molecular biology. Furthermore, laser light is used for a variety of experiments because it can be better focused compared to other light sources; it is monochromatic, and very short pulses can be produced.

A Free Electron Laser (FEL) produces laser radiation with tunable wavelength. At the German Electron Synchrotron (DESY) in Hamburg the X-ray Free Electron Laser research project XFEL is conducted. The goal of the project is to build a Free Electron Laser operating in the X-ray wavelength by the year 2012 [1].

The process uses a linear particle accelerator, which increases the energy of electrons by interaction with electromagnetic radio frequency (RF) fields to a desired value. These fields are required to follow a reference with very small amplitude as well as phase errors. Figure 2 shows the structure of the Vacuum Ultraviolet FEL (FLASH) which is already working at DESY. The linear accelerator consists of resonators for the RF-fields housed in cryomodules. The RF-fields inside these superconducting resonator cavities are supplied by an actuator system for a finite time interval and then turned off again periodically.

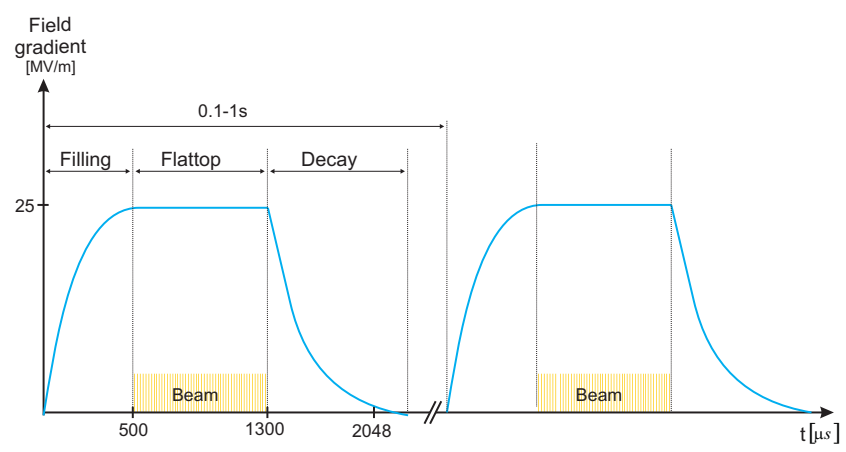

Fig. 1: One RF-pulse in superconducting cavities

In Figure 1 the amplitude of the desired envelope of the RF-field is displayed for one RF-pulse as a function of time. The field inside the accelerator cavities has to be kept constant once the required amplitude for the appropriate energy gain of the electrons has been reached at the end of the so called filling phase. During the flat top phase the electron beam is injected into the accelerator. When the electron beam has passed, the RF-field is turned off and the field amplitude decays. The envelope of the RF-field oscillation must be kept constant in amplitude and phase during the flat top time interval to transfer a precise amount of energy to the electrons. 


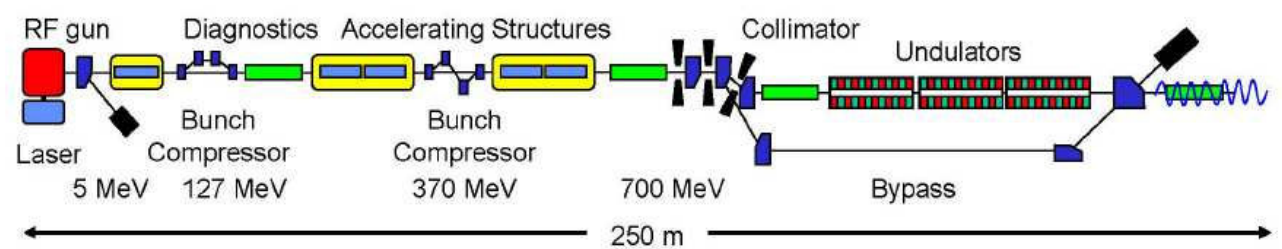

Fig. 2: A Free Electron laser with front end, accelerating structures and undulators

Once the system is set up to a desired operating point, the reference signal remains unchanged for a large number of pulses. Therefore repetitive disturbances can be suppressed by finding an optimal feedforward control signal to minimize the deflection from the reference. The adaption of the driving signal is calculated by using an iterative learning control algorithm proposed in [4]. Further information can be found in [5], [6], and [7]. For this procedure as for feedback controller design a good model of the system dynamics is needed. Therefore black box models have been identified.

\section{B. RF System of the Linear Accelerator}

The acceleration takes part in the resonators where standing RF-waves (modes) provide the energy. The resonance frequency of $1.3 \mathrm{GHz}$ for the desired acceleration mode is determined by the geometry. If the length of the cavity changes, the resonance frequency changes as well. Due to the relatively thin walls, the resonators become susceptible to mechanical vibrations called microphonics which detune the resonance frequency. The high power RF-fields in the cavities lead to deformation of the cavity walls and therefore detuning as well. Induced currents cause Lorentz forces acting on the metal surroundings during the pulse sequence. Measurements have shown that detuning can result in a change up to $\Delta f \approx 500 \mathrm{~Hz}$ of the resonance frequency. Since the Lorentz force is induced every time the electric field is generated, the Lorentz force detuning is considered deterministic and repetitive.

Another source of disturbance is the electron beam itself. While it is passing the accelerating structure, the charged particles gain energy from the present RF-field which leads to fluctuations in the present amount of energy stored in the system. The following bunches of charged particles will be influenced by these fluctuations, which have to be minimized by the control system. One can assume that the bunch arrival time will be constant from pulse to pulse, thus having the properties of a repetitive disturbance.

\section{Digital Control System}

The actuator system receives a precise RF signal of $1.3 \mathrm{GHz}$ from the master oscillator (MO). This low power sinusoidal signal can be changed by the vector modulator in amplitude and phase. The output signal of the vector modulator is amplified by a klystron, which is a radio frequency amplifier. The amplified RF waves are transferred from the klystron to the cavities inside the cryomodules via a waveguide transmission and distribution system. For economical reasons, one high power klystron supplies all $8-32$ cavities of an RF station, thus RF fields can not be influenced in each cavity individually - the system is underactuated.

The superconduction cavity simulator and controller (SIM$\mathrm{CON}$ ) is based on field programmable gate array (FPGA) structures. It allows the implementation of fast algorithms. A block diagram of the Low Level Radio Frequency (LLRF) control system is presented in Fig. 3, where the lower part shows the digital FPGA controller. The LLRF control system has the task of keeping the pulsed RF fields in the superconducting cavities of the RF station at the reference value during the flat top phase of one RF pulse shown in Figure 1.

After measuring the actual RF-field by pickup antennas, the signals are downconverted to an intermediate frequency of $250 \mathrm{kHz}$. The real (I) and imaginary (Q) field measurement signals go through analog-digital-converters (ADC) with a sampling frequency of $1 \mathrm{MHz}$. An overview of the signals shown in Fig. 3 is represented in terms of $I$ and $Q$ components.

- Input signals $u_{\mathrm{I}}, u_{\mathrm{Q}}$ : Control signals of the actuator system are directly acting on the vector modulator.

- Output signals $y_{\mathrm{I}}, y_{\mathrm{Q}}$ : The real and imaginary part of the sum of the RF-field voltage vectors of eight cavities.

- Reference signals $r_{\mathrm{I}}, r_{\mathrm{Q}}$ : Reference signals of the real and imaginary part of the vector sum of the RF-field's voltage vectors given by look-up tables for the specified field gradient.

- Feedforward signals $f_{\mathrm{I}}, f_{\mathrm{Q}}$ : Part of the control signals determined by open loop control.

- Control signals $u_{\mathrm{c}, \mathrm{I}}, u_{\mathrm{c}, \mathrm{Q}}$ : The learning controller output signals, updating the previous iteration input signals

- Control error signals $e_{\mathrm{I}}, e_{\mathrm{Q}}$ : Deviations in real and imaginary part of the output signals from the reference signals.

A calibration of the measurement signals is done for compensation of effects resulting e.g. from different cable lengths. The control algorithm usually uses the vector sums of all calibrated measurement signals of the individual cavities as signals to be controlled, because of the lack of individual actors for each cavity.

The desired setpoint could even be reached with pure feedforward control if the input signals are sufficiently adapted to the disturbance sources. Strong disturbances are caused by the Lorentz forces which are deterministic from pulse to 


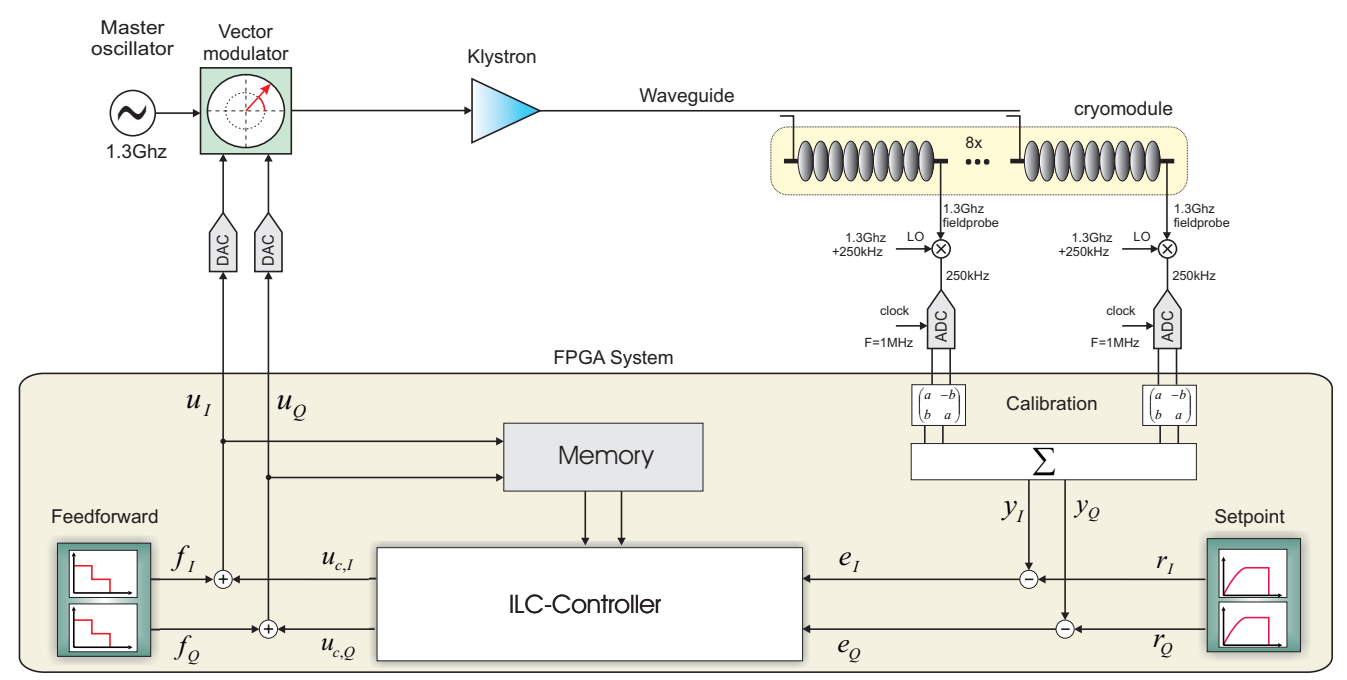

Fig. 3: Structure of the RF system with master oscillator, vector modulator, klystron, cryomodule, measurement and calibration system and the FPGA implemented control system

pulse. It is possible to compensate these by smooth changes of the input signal over the flattop. Moreover, transients induced by the beam are predictable, and the arrival time is known. An increase in the driving power will keep these fluctuations low. Both compensations have a positive effect on a feedback control performance by keeping the control error signals small.

To predict the system behavior, it is essential to have an adequate model of the underlying system dynamics. The following section gives a brief outline of the identification procedure which was used for modelling.

\section{SYSTEM MODELING}

Although additional external disturbances and a number of nonlinearities in the actuator system are known to be relevant for a broad range of operation setpoints, standard identification procedures for linear time invariant (LTI) models could be used to estimate black box models that can be validated at specific setpoints [13]. The subspace identification method N4SID, provided by Matlab's System Identification Toolbox [15] is used to estimate parameters $A, B, C, D$ of a black box state space model

$$
\begin{aligned}
& \dot{x}(t)=A x(t)+B u(t), \\
& y(t)=C x(t)+D u(t),
\end{aligned}
$$

where $u=\left(\begin{array}{ll}u_{I} & u_{Q}\end{array}\right)^{T}$ and $y=\left(\begin{array}{ll}y_{I} & y_{Q}\end{array}\right)^{T}$ denote the system input and output vector, respectively and $x$ is the state vector of the system. The flat top phase of the pulse is of main interest for control and is at the same time marking the systems operation point. Only measurements from this period are used for system identification. Persistent excitation signals can be injected into the accelerator system by superimposing random signals on standard feedforward tables with defined setpoints. A typical input sequence for the feedforward table is shown in Fig. 4.

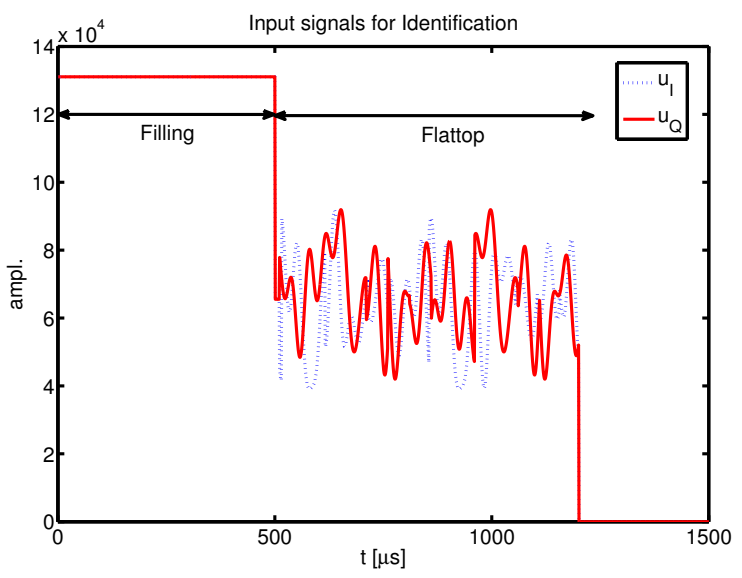

Fig. 4: Input Disturbances on both channels during flat top

In the first $500 \mu$ s (filling phase), the actuator system is operated at maximum power. In the flat top phase starting from $500 \mu \mathrm{s}$, the inputs are first reduced by a factor of 0.5 to reach the setpoint and soon after, the zero mean excitation signal is added to both inputs, see Fig. 4. A high amplitude leads to a good signal to noise ratio. In Fig. 5 measured vs. simulated signals are shown for an identified 3rd order model.

An investigation of the system properties as well as the disturbances motivated the idea to use an Iterative Learning Controller (ILC) in order to increase the performance of the system during the flat top phase.

\section{ITERATIVE LEARNING CONTROL}

Iterative Learning Control (ILC) is used for repetitive processes consisting of a number of trials each representing the same procedure. Since the system to be controlled is driven in pulsed mode, the accelerating process is considered to be repetitive. Moreover, the disturbances show this behavior as 

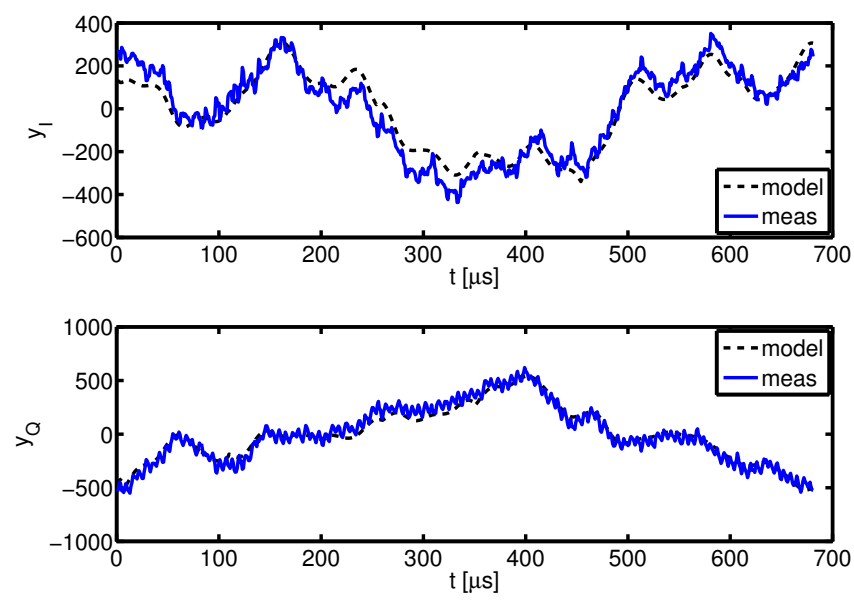

Fig. 5: Measured vs. simulated vector sum signals (3rd order model), flattop phase

well.

An ILC uses measures of the performance of previous trials in order to improve the next trial by updating the inputs. The objective of an ILC can be described by

$$
\left\|e_{k}\right\| \rightarrow 0 \text { as } k \rightarrow \infty, k \in \mathrm{N}
$$

where $e_{k}=\left(e_{I} e_{Q}\right)^{T}$ denotes the tracking error vector signal of the desired trajectory of the $k$ th trial.

One recently proposed iterative learning control algorithm is the Fast-Norm-Optimal Iterative Learning Controller (FNOILC), which uses the system model in a state space representation [4]. The small number of computations between the trials is favorable for the given hardware setup. In order to be able to compute an optimal input signal, a criterion has to be determined that reflects the goal of the control task and thus, the quality of the input. This is done by solving the following minimum-norm optimization problem:

$u_{k+1}=\arg \min _{u_{k+1}}\left\{J_{k+1}\left(u_{k+1}\right): e_{k+1}=r-y_{k+1}, y_{k+1}=G u_{k+1}\right\}$,

with the performance index

$$
J_{k+1}\left(u_{k+1}\right)=\left\|e_{k+1}\right\|_{Y}^{2}+\left\|u_{k+1}-u_{k}\right\|_{U}^{2},
$$

where the norms $\|\cdot\|$ are defined as $\|u\|_{U}^{2}=u^{T} W_{2} u$ and $\|y\|_{Y}^{2}=y^{T} W_{1} y$ for the input and output spaces $\mathbf{U}$ and $\mathbf{Y}$, respectively. The system model is estimated during the identification procedure and represented as matrix

$$
G=\left[\begin{array}{cccc}
C B & 0 & \cdots & 0 \\
C A B & C B & \cdots & 0 \\
\vdots & \vdots & \ddots & \vdots \\
C A^{N-1} B & C A^{N-2} B & \cdots & C B
\end{array}\right] .
$$

The performance criterion described in (3) can be rewritten as

$$
\begin{aligned}
J_{k+1} & =\sum_{t=1}^{N}\left[r(t)-y_{k+1}(t)\right]^{T} W_{1}(t)\left[r(t)-y_{k+1}(t)\right]+ \\
& +\sum_{t=0}^{N-1}\left[u_{k+1}(t)-u_{k}(t)\right]^{T} W_{2}(t)\left[u_{k+1}(t)-u_{k}(t)\right] .
\end{aligned}
$$

TABLE I: Control Algorithm F-NOILC

$$
\begin{aligned}
& \text { First level (before operation): } \\
& K(t)=A^{T} K(t+1) A+C^{T} W_{1}(t+1) C \\
& -\left[A^{T} K(t+1) B\left\{B^{T} K(t+1) B+W_{2}(t+1)\right\}^{-1}\right. \\
& \left.B^{T} K(t+1) A\right] \\
& \alpha(t)=\left\{I+K(t) B W_{2}^{-1}(t) B^{T}\right\}^{-1} \\
& \beta(t)=\alpha(t) A^{T} \\
& \gamma(t)=\alpha(t) C^{T} W_{1}(t+1) \\
& \omega(t)=W_{2}^{-1}(t) B^{T} \\
& \lambda(t)=\quad\left(B^{T} K(t) B+W_{2}(t)\right)^{-1} B^{T} K(t) A \\
& \xi_{k+1}(t)=\beta(t) \xi_{k+1}(t+1)+\gamma(t) e_{k}(t+1)
\end{aligned}
$$

Third level (between sampling instants):

$$
u_{k+1}(t)=u_{k}(t)-\lambda(t)\left\{x_{k+1}(t)-x_{k}(t)\right\}+\omega(t) \xi_{k+1}(t)
$$

The quantities $W_{1}(t)$ and $W_{2}(t)$ represent time varying weighting matrices which have to be symmetric and positive definite for all $t$. The $(k+1)$ th trial control input is determined such that it reduces the tracking error $e$ while keeping the deviation from the control input used in the $k$ th trial small. The F-NOILC algorithm is given in Table I.

The algorithm is divided into three levels. In the first level the algorithm is initialized. In the second level computations are carried out between trials, such as the calculation of a predictive component. The input signals with a state feedback component are computed in the third level, which represents computations between the sampling instants of a trial.

\section{A. Simulations}

Fast and efficient computation is important when computing the updated input signal before the next trial starts. The time interval between two consecutive RF-pulses is approximately $0.1 s$, where the computed update of the input signal must be transferred into the FPGA. At this moment a state feedback controller is not implemented on the real machine. Therefore the system model is used to estimate the states before the next trial starts, e.g the third level computations are done in the second level. Small model variations in this have a strong influence on the performance of the algorithm. To overcome this drawback in future an output feedback scheme will be used.

In order to ensure that system components are not damaged when the algorithm is implemented on the real plant, the input signals are limited as shown in Fig. 6. The limits are set to the maximum and minimum values of the input signals during the filling and the decay phase.

The following weighting matrices led to satisfactory results:

$$
W_{1}=100 \times I^{2 \times 2} \text { and } W_{2}=I^{2 \times 2} .
$$

For the simulation input and output disturbances are used to model the influences of the electron beam on the real machine, which is the main source of repetitive disturbance 

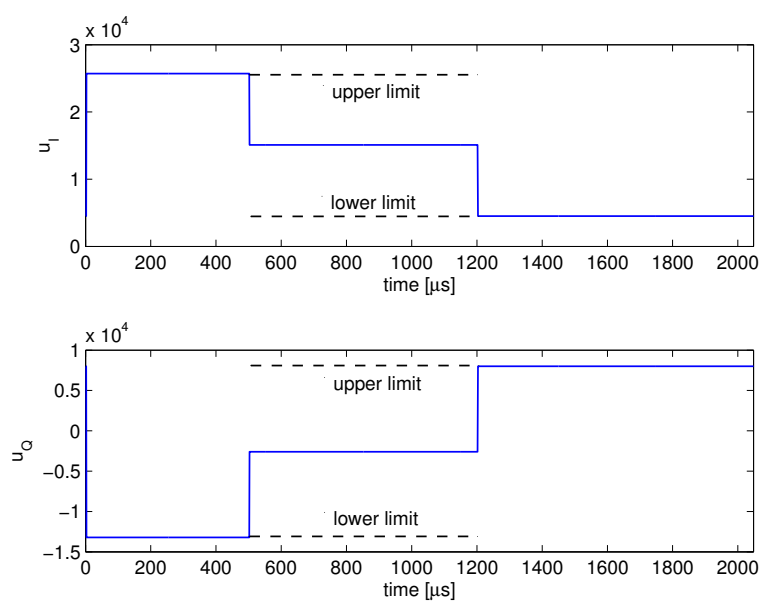

Fig. 6: Limits for input signals

to be controlled. The results for the flat top phase are shown in Fig. 7. As the number of trials increases, the output signals approach the desired setpoint (SP) trajectory. Rejection of the included input and output disturbances can also be observed. Since the input signal reaches the given limits in the beginning of the phase, the output signal only approaches the setpoint slowly in the first $100 \mu \mathrm{s}$.

Convergence speed and long time stability of the ILC
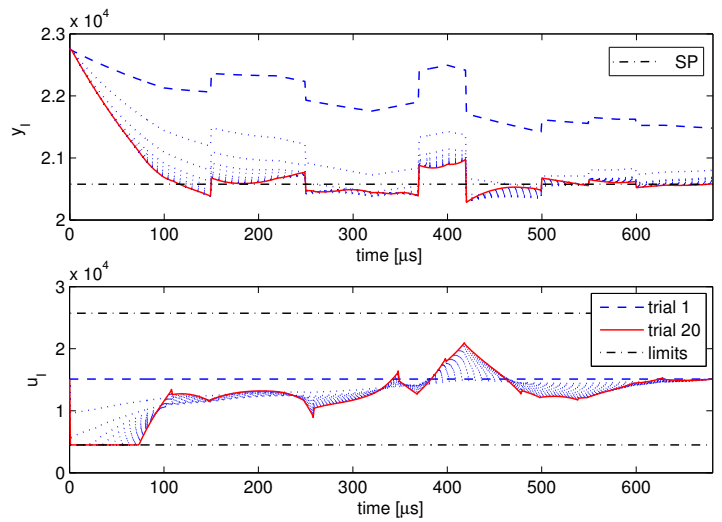

Fig. 7: Output and input signals after 20 iterations

is very important for realtime user operation. Therefore the algorithm is tested on the real plant in presence of measurement disturbances and possible plant variations.

\section{EXPERIMENTAL RESUlts}

The F-NOILC was successfully implemented on the real plant at the DESY test facility. The results are shown in Fig. 8 to 10 . The state variables required for the state feedback component are computed using the identified model of the plant,as already described before. The weighting matrices are set to the values in (6). Because of the large computation time the input signals are computed for a whole trial at each iteration. The number of iterations is set to 10 .
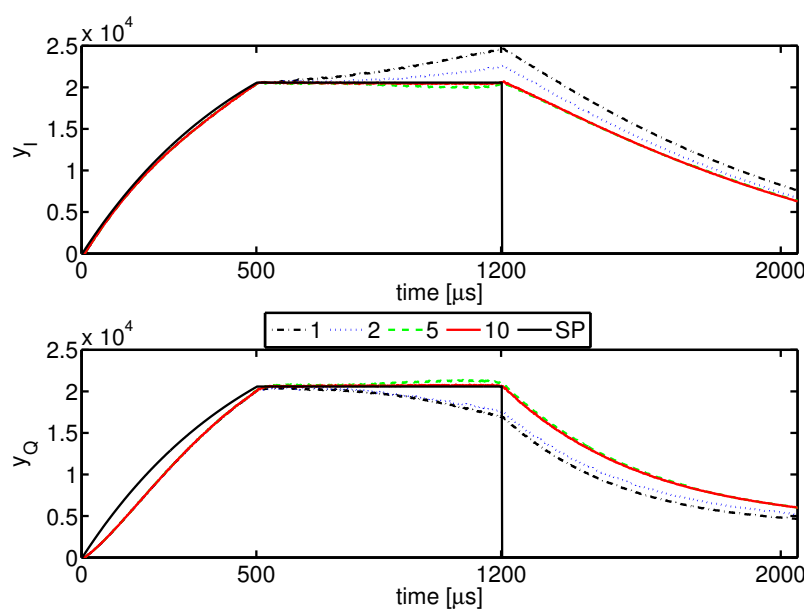

Fig. 8: Measured output signals
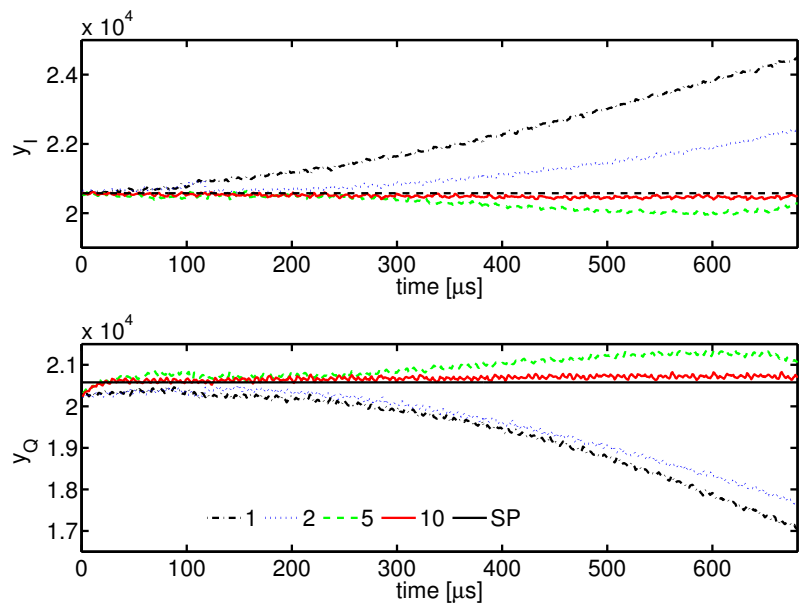

Fig. 9: Measured output signals (flat top phase)

Fig. 8 and Fig. 9 show an increasing and decreasing trend of the first and the second output signal during the flat top phase, respectively. This is the general behavior caused by the detuning effects described before. However, increasing the number of trials, both output signals approach the desired setpoint. After 10 iterations, the output signals show only small deviations from the reference trajectory. Since only the signals during the flat top phase are controlled, the input signals of the filling and the decay phase are kept constant as illustrated in Fig. 10.

Considering the output signals $y_{I}$ and $y_{Q}$ as the real and imaginary part of an output vector, respectively, the amplitude and phase of the vector can be computed. In order to evaluate the performance of the F-NOILC, the peak-to-peak error of the amplitude $(A)$ is computed as follows:

$$
e_{A, p 2 p}=\frac{\left\|\max \left(e_{A}(t)\right)-\min \left(e_{A}(t)\right)\right\|}{\left\|\operatorname{setpoint}_{A}\right\|},
$$

with $e_{A}(t)=y_{A}(t)-r_{A}(t)$, where $y(t)$ and $r(t)$ are the output signals and the reference signals, respectively. 

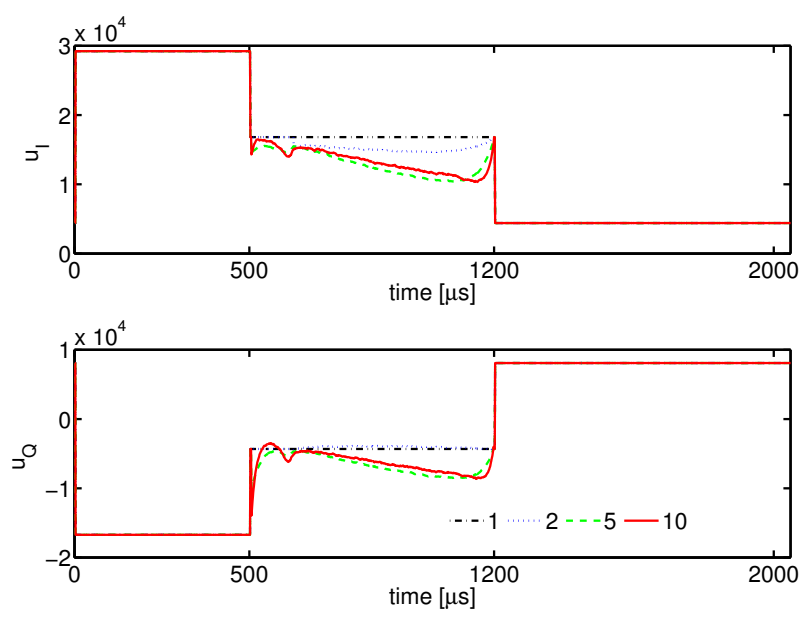

Fig. 10: Input signals

The RMS-error is computed as

$$
e_{A, r m s}=\sqrt{\frac{1}{T_{f t}} \sum_{k=500}^{1200} e_{A}^{2}(k)}
$$

with $t_{0, f t}$ defining the beginning of the flat top phase and $T_{f t}$ the time interval of the flat top phase. For the phase $(\phi)$ the errors are computed similarly. The results are given in Table II.

TABLE II: Performance objectives and performance achieved with F-NOILC

\begin{tabular}{|c||c|c||c|c|}
\hline \multicolumn{1}{|c||}{} & \multicolumn{2}{c||}{ Amplitude $A$} & \multicolumn{2}{c|}{ Phase $\phi$} \\
\hline \hline & $\mathbf{e}_{\mathbf{A}, \mathbf{p 2 p}}$ & $\mathbf{e}_{\mathbf{A}, \mathbf{r m s}}$ & $\mathbf{e}_{\phi, \mathbf{p} 2 \mathbf{p}}$ & $\mathbf{e}_{\phi, \mathbf{r m s}}$ \\
\hline F-NOILC & $1.06 \%$ & 31.76 & $2.42 \%$ & $0.2879^{\circ}$ \\
\hline P-controller & $0.5 \%$ & 0.1 & $1 \%$ & $0.1^{\circ}$ \\
\hline Objectives & $\leq 0.1 \%$ & $\leq 0.01$ & $\leq 0.1 \%$ & $\leq 0.01^{\circ}$ \\
\hline
\end{tabular}

For comparison, the defined objectives as well as the performance achieved using the already implemented proportional feedback controller are given in the table. It can be seen that the performance of the F-NOILC does not reach the given objectives in open loop without realtime feedback [8]. However, the tracking errors of the F-NOILC after only 10 iterations are close to the values that were achieved with the proportional controller and closed to the desired values defined by the objectives. In future a combination of a realtime feedback in combination with the ILC algorithm has to be studied as well.

\section{CONCLUSIONS AND FUTURE WORKS}

An Iterative Learning Controller (ILC) has been used to improve the system performance of the accelerator. A FastNorm-Optimal Iterative Learning Controller (F-NOILC) was selected and its properties were investigated. First, a simulation using a model obtained by system identification was executed. After analyzing the results, the F-NOILC was adapted by changing the realtime state feedback to an model estimated input computation, in order to make it possible to apply the controller to the real plant.

The adapted F-NOILC was successfully implemented at the FLASH and first tests were run. Experimental results were presented which show performance improvements of the system to be controlled. Even though properties of the control algorithm had to be changed and only tests with a small number of iterations could be executed, the ILC achieved good results.

So far it has not been possible yet to investigate the performance of the controller when an electron beam is injected into the system - experiments including the beam loading will be carried out in the future, showing the importance of the adaption of the feedforward signals, due to the fact that the feedback itself is not able to compensate for this repetitive disturbances. For further improvement of the performance, tests with an increased number of iterations will be performed to confirm long term stability. Moreover, implementing the control algorithm in the FPGA will decrease the computational time needed so that input signals can be computed in every sampling period. A combination of the ILC with the existing proportional controller or a MIMOLTI controller which is currently being developed [16], is expected to further increase the control performance [11].

\section{REFERENCES}

[1] Brinkmann, R. et al., TESLA XFEL Technical Design Report, DESY, Hamburg, 2002

[2] Bristow, D. A.; Tharayil, M.; Alleyne, A. G. A Survey of Iterative Learning Control A learning-based method for high-performance tracking control, IEEE Control Systems Magazine, Vol. 26, pages 96-114, 2006.

[3] Moore, K.L.; YangQuan Chen; Hyo-Sung Ahn, Iterative Learning Control: A Tutorial and Big Picture View 45th IEEE Conference on Decision and Control, pp. 2352-2357, 2006

[4] Ratcliffe, J.D. et al.,Norm-Optimal Iterative Learning Control Applied to Gantry Robots for Automation Applications, IEEE Transactions on Robotics, Vol. 22,No. 6, 2006

[5] Hwang, D.-H., BIEN, Z., Oh, S.-R.Iterative Learning Control method for discrete time systems, IEEE Proc. D, 1991

[6] Ratcliffe J.D. et al.,P-type iterative learning control for systems that contain resonance, Int. J. Adapt. Control Signal Process, 2005

[7] Arimoto S. et al.,Bettering operation of robots by learning, J. Robot Syst., 1984

[8] Amann N. et al.,Iterative learning control using optimal feedback and feedforward actions, Int. J. Control,1996

[9] Kailath T.,Linear Systems, Prentice Hall, 1980

[10] Anderson, B.D.O and Moore J.B.,Optimal Control - linear optimal control, Prentice Hall, 1989

[11] Owens, D.H.,Iterative learning control: convergence using high gain feedback, Proceedings on 31st IEEE Conference on Decision on control, 1992

[12] Liepe, M. et al., Dynamic Lorentz Force Compensation with a Fast Piezoelectric Tuner, TESLA Note 2001-3, Hamburg, 2001

[13] Ljung, Lennart, System identification - Theory for the user, Prentice Hall, New Jersey, 1999

[14] Schilcher, Thomas, Vector sum control of pulsed accelerating fields in lorentz force detuned superconducting cavities, Phd-Thesis, University of Hamburg, Germany, 1998

[15] The Mathworks, System Identification Toolbox User's Guide, The Mathworks, Inc., Natick, 2004

[16] Schmidt, C. et al., Automatic Tuning of the first Multivariable Low Level RF Controller with FPGA technique for the Free Electron Laser FLASH, American Control Conference, 2008 